\title{
Increase in the Accuracy of the Parameters Identification for a Vibrating Ring Microgyroscope Operating in the Forced Oscillation Mode with Nonlinearity Taken into Account
}

\author{
D. A. Maslov, I. V. Merkuryev
}

The dynamics of a vibrating ring microgyroscope operating in the forced oscillation mode is investigated. The elastic and viscous anisotropy of the resonator and the nonlinearity of oscillations are taken into consideration. Additional nonlinear terms are suggested for the mathematical model of resonator dynamics. In addition to cubic nonlinearity, nonlinearity of the fifth degree is considered. By using the Krylov-Bogolyubov averaging method, equations containing parameters characterizing damping, elastic and viscous anisotropy, as well as coefficients of oscillation nonlinearity are deduced. The parameter identification problem is reduced to solving an overdetermined system of algebraic equations that are linear in the parameters to be identified. The proposed identification method allows testing at large oscillation amplitudes corresponding to a sufficiently high signal-to-noise ratio. It is shown that taking nonlinearities into account significantly increases the accuracy of parameter identification in the case of large oscillation amplitudes.

Keywords: parameter identification, vibrating ring microgyroscope, nonlinear oscillations

Received May 08, 2018

Accepted June 28, 2018

This work was supported by the Russian Foundation for Basic Research (projects nos. 16-01-00772-a, 16-08-01269-a).

Dmitry A. Maslov

MaslovDmA@mpei.ru

Igor V. Merkuryev

MerkuryevIV@yandex.ru

National Research University "Moscow Power Engineering Institute"

ul. Krasnokazarmennaya 14, Moscow, 111250, Russia

RUSSIAN JOURNAL OF NONLINEAR DYNAMICS, 2018, 14(3), 377-386 


\section{Introduction}

Nowadays the vibrating microgyroscopes are one of the most promising inertial sensors used in navigation systems for various vehicles [1]. In this paper, the vibrating ring microgyroscope is considered as a micromechanical realization of the wave solid-state gyroscope with a ring resonator.

The foundations of the theory of a wave solid-state gyroscope have been laid down in [2] and [3]. Issues of instrumental error identification and methods of increasing accuracy were considered in [3-8]. In these studies, linear equations of small resonator oscillations were used for parameter identification. Phenomena such as oscillation amplitude disruption inherent to nonlinear systems were detected during experimental studies of the dynamics of vibrating gyroscopes [9-11]. The impact of nonlinearity on the dynamics of micromechanical and wave solid-state gyroscopes is studied in detail in [7]. Nonlinearity can be neglected only at small oscillation amplitudes with a rather low signal-to-noise ratio. To increase this ratio, the amplitude of oscillations should be increased, but in this case the errors caused by nonlinearity start to grow too. To take nonlinearity into account in the parameter identification process, a special method was developed to identify the coefficient of cubic nonlinearity simultaneously with other parameters of the mathematical model of gyroscope resonator oscillations [12]. This method made it possible to significantly improve the accuracy of identification of all parameters and to identify the value of the cubic nonlinearity coefficient for the mathematical model. However, further increase in the amplitude requires refining the nonlinear mathematical model. Therefore, the goal of this paper is to develop a parameter identification method by using additional nonlinear terms in the mathematical model.

\section{Resonator oscillation equations}

We consider the gyroscope with a ring resonator shown in Fig. 1. The ring resonator 2 is connected to the base 1 by torsion bars 3 (Fig. 1). The resonator midline in undeformed state is a circle of radius $R$. Resonator oscillations are excited and measured by the system of control and measuring electrodes, respectively. Each contour is formed by two nearest torsion bars and a section of a resonator with a circumferential size of $\pi / 4$.

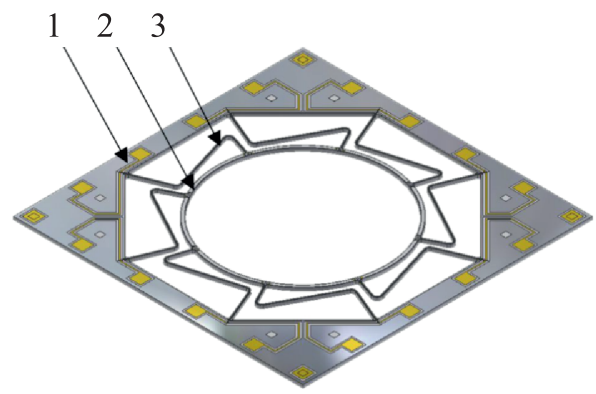

Fig. 1. Ring microgyroscope.

The second basic mode of oscillations for a thin elastic ring resonator is the superposition of two normal modes of oscillations that are turned relative to each other by the angle $\pi / 4$. The primarily normal mode of resonator oscillations is excited by the control loop system. Due to rotation of the gyroscope base about the axis, perpendicular to the plane of the ring resonator, 
the secondary normal mode of oscillations arises. Antinodes of the secondary normal mode of oscillations are nodes of the primarily normal mode of oscillations.

Nonlinear differential equations describing the dynamics of the ring resonator $[4,7]$ can be written as follows:

$$
\begin{aligned}
& \ddot{f}+\omega^{2} f=-\left(c+h_{c}\right) f-\left(n+h_{s}\right) g-\left(\gamma+b_{c}\right) \dot{f}-\left(v+b_{s}\right) \dot{g}-u_{1} \sin \omega_{0} t+u_{2} \cos \omega_{0} t+F_{1}, \\
& \ddot{g}+\omega^{2} g=-\left(c-h_{c}\right) g-\left(-n+h_{s}\right) f-\left(\gamma-b_{c}\right) \dot{g}-\left(-v+b_{s}\right) \dot{f}-u_{3} \sin \omega_{0} t+u_{4} \cos \omega_{0} t+F_{2},
\end{aligned}
$$

where $f$ and $g$ are generalized coordinates of the second fundamental mode of resonator oscillations that are taken at two fixed points spaced apart from each other at angle $\pi / 4 ; \omega$ is the characteristic frequency of the resonator; $c$ and $n$ are parameters of positional forces; $h_{s}=h \sin 4 \alpha$, $h_{c}=h \cos 4 \alpha$ and $b_{s}=b \sin 4 \beta, b_{c}=b \cos 4 \beta$ are components describing elastic and viscous anisotropy, respectively, $h$ and $b$ are elastic and viscous anisotropy modules, $\alpha$ and $\beta$ are orientation angles of the principal axis of stiffness and dissipation relative to the reference axes; $\gamma$ is the damping factor; $v$ is the parameter characterizing the angular velocity; $u_{1}, u_{2}, u_{3}, u_{4}$ are normalized amplitudes of the resonator oscillations driving signals; and $\omega_{0}$ is the frequency of the external harmonic excitation.

Methods to identify the coefficients of dynamics equations for the ring resonator are presented in $[4,8]$ without allowance for nonlinearity and in [12] with the coefficient of cubic nonlinearity taken into account. When considering the supply of voltage to the oscillation control electrodes, cubic nonlinearity was deduced in [13], with higher-order nonlinearities being neglected. To increase the accuracy of parameter identification, we consider equations (1), which include nonlinearities of the fifth degree.

Thus, we consider the following cases:

1) cubic nonlinearity of spherical type

$$
F_{1}=\xi\left(f^{2}+g^{2}\right) f, \quad F_{2}=\xi\left(f^{2}+g^{2}\right) g,
$$

2) cubic and fifth degree nonlinearities of spherical type

$$
\begin{aligned}
& F_{1}=\xi\left(f^{2}+g^{2}\right) f+\eta\left(f^{2}+g^{2}\right)^{2} f, \\
& F_{2}=\xi\left(f^{2}+g^{2}\right) g+\eta\left(f^{2}+g^{2}\right)^{2} g,
\end{aligned}
$$

3) the linear model with $F_{1}=0, F_{2}=0$ to be used for comparison.

We note that the linear mathematical model of gyroscope resonator oscillations which is obtained by substitution $F_{1}=F_{2}=0$ into Eqs. (1) contains 12 parameters to be identified. The mathematical model including cubic nonlinearity $(\eta=0)$ has 13 parameters. The mathematical model including cubic and fifth degree nonlinearities has 14 parameters.

\section{Averaging of the motion equations}

We use the Krylov - Bogolyubov averaging method [14] to investigate the resonator dynamics under the soft resonance excitation of oscillations. Equations (1) are examined.

The frequency of the external excitation is assumed to be close to the resonant frequency of the resonator oscillations

$$
\omega_{0}=\omega+\lambda, \quad \lambda \ll \omega,
$$

where $\lambda$ is the frequency adjustment introduced for tuning the gyroscope operation near the resonance. 
The solution of the system (1) has an oscillatory character with a resonant frequency, $\omega$, of about $10 \mathrm{kHz}$. In the absence of perturbations on the right-hand side of (1), the solution of the system (1) is purely harmonic. Small perturbations are caused by instrumental errors involved in manufacturing the resonator, by distinctions in damping of oscillations, gyroscopic forces and nonlinearity inherent in gyroscopes of the Foucault generalized pendulum class [3]. Small positional and velocity forces on the right-hand side of Eqs. (1) lead to a small change in the resonant frequency by approximately $1 \mathrm{~Hz}$, which allows one to apply asymptotic methods for investigating the problem. We apply the Krylov-Bogolyubov averaging method to the system (1). Previously the system (1) is reduced to the standard form by conversion variables $f, \dot{f}, g, \dot{g}$ to homogenized slow variables $p_{1}, q_{1}, p_{2}, q_{2}$ as follows:

$$
\begin{array}{ll}
f=p_{1} \sin \omega_{0} t+q_{1} \cos \omega_{0} t, & \dot{f}=p_{1} \omega_{0} \cos \omega_{0} t-q_{1} \omega_{0} \sin \omega_{0} t \\
g=p_{2} \sin \omega_{0} t+q_{2} \cos \omega_{0} t, & \dot{g}=p_{2} \omega_{0} \cos \omega_{0} t-q_{2} \omega_{0} \sin \omega_{0} t .
\end{array}
$$

Substituting (2) into (1), resolving the obtained system of equations with respect to derivatives of slow variables and averaging over the explicitly contained time $[1,7]$, we deduce a system of dynamic equations in the slow variables $p_{1}, q_{1}, p_{2}, q_{2}$ as the first approximation of the averaging method. The deduced system can be written in matrix form as follows:

$$
\begin{gathered}
\dot{\mathbf{q}}=D \mathbf{z}-\mathbf{y}, \\
\mathbf{q}=\left(q_{1}, p_{1}, q_{2}, p_{2}\right)^{T}, \\
\mathbf{y}=\lambda\left(p_{1},-q_{1}, p_{2},-q_{2}\right)^{T},
\end{gathered}
$$

where the blocks of the matrix $D$ and the parameters vector $\mathbf{z}$ have the form

$$
\begin{gathered}
D=\left(D_{\gamma} D_{\xi}\right), \quad \mathbf{z}=\left(\mathbf{z}_{\gamma}^{T} \mathbf{z}_{\xi}^{T}\right)^{T}, \\
D_{\gamma}=\frac{1}{2} \cdot\left(\begin{array}{cccccccccccc}
-q_{1} & -q_{2} & -q_{1} & -q_{2} & p_{1} & p_{2} & p_{1} & p_{2} & 1 & 0 & 0 & 0 \\
-p_{1} & -p_{2} & -p_{1} & -p_{2} & -q_{1} & -q_{2} & -q_{1} & -q_{2} & 0 & 1 & 0 & 0 \\
-q_{2} & q_{1} & q_{2} & -q_{1} & p_{2} & -p_{1} & -p_{2} & p_{1} & 0 & 0 & 1 & 0 \\
-p_{2} & p_{1} & p_{2} & -p_{1} & -q_{2} & q_{1} & q_{2} & -q_{1} & 0 & 0 & 0 & 1
\end{array}\right), \\
\mathbf{z}_{\gamma}=\left(\gamma, v, b_{c}, b_{s}, \widetilde{c}, \widetilde{n}, \widetilde{h}_{c}, \widetilde{h}_{s}, \widetilde{u}_{1}, \widetilde{u}_{2}, \widetilde{u}_{3}, \widetilde{u}_{4}\right)^{T},
\end{gathered}
$$

where $\widetilde{c}=c / \omega, \widetilde{n}=n / \omega, \widetilde{h}_{c}=h_{c} / \omega, \widetilde{h}_{s}=h_{s} / \omega, \widetilde{u}_{i}=u_{i} / \omega, i=1,2,3,4$ and also $\widetilde{\xi}=\xi / \omega$, $\widetilde{\eta}=\eta / \omega$.

1) For cubic nonlinearity of spherical type

$$
D_{\xi}=\left(\begin{array}{c}
k_{1} \\
k_{2} \\
k_{3} \\
k_{4}
\end{array}\right), \quad \mathbf{z}_{\xi}=\widetilde{\xi},
$$

where $k_{1}=-p_{1} E-q_{2} K, k_{2}=q_{1} E-p_{2} K, k_{3}=-p_{2} E+q_{1} K, k_{4}=q_{2} E+p_{1} K, E=3\left(q_{1}^{2}+p_{1}^{2}+\right.$ $\left.+q_{2}^{2}+p_{2}^{2}\right) / 8, K=\left(p_{2} q_{1}-p_{1} q_{2}\right) / 4$. 
2) For cubic and fifth degree nonlinearities of spherical type

$$
D_{\xi}=\left(\begin{array}{cc}
k_{1} & k_{5} \\
k_{2} & k_{6} \\
k_{3} & k_{7} \\
k_{4} & k_{8}
\end{array}\right), \quad \mathbf{z}_{\xi}=(\widetilde{\xi}, \widetilde{\eta})^{T}
$$

where

$$
\begin{aligned}
k_{5} & =-\frac{1}{16}\left(5 p_{1}^{5}+12 p_{1}^{2} p_{2} q_{1} q_{2}+4 p_{2} q_{1} q_{2}\left(p_{2}^{2}+q_{1}^{2}+q_{2}^{2}\right)+2 p_{1}^{3}\left(5\left(p_{2}^{2}+q_{1}^{2}\right)+q_{2}^{2}\right)+\right. \\
& \left.+p_{1}\left(5 p_{2}^{4}+6 p_{2}^{2} q_{1}^{2}+5 q_{1}^{4}+6\left(p_{2}^{2}+q_{1}^{2}\right) q_{2}^{2}+q_{2}^{4}\right)\right), \\
k_{6} & =\frac{1}{16}\left(5 p_{1}^{4} q_{1}+4 p_{1}^{3} p_{2} q_{2}+4 p_{1} p_{2} q_{2}\left(p_{2}^{2}+3 q_{1}^{2}+q_{2}^{2}\right)+2 p_{1}^{2} q_{1}\left(3 p_{2}^{2}+5 q_{1}^{2}+3 q_{2}^{2}\right)+\right. \\
& \left.+q_{1}\left(p_{2}^{4}+5\left(q_{1}^{2}+q_{2}^{2}\right)^{2}+2 p_{2}^{2}\left(q_{1}^{2}+3 q_{2}^{2}\right)\right)\right), \\
k_{7} & =-\frac{1}{16}\left(p_{2}\left(5\left(p_{1}^{2}+p_{2}^{2}\right)^{2}+2\left(3 p_{1}^{2}+p_{2}^{2}\right) q_{1}^{2}+q_{1}^{4}\right)+4 p_{1} q_{1}\left(p_{1}^{2}+3 p_{2}^{2}+q_{1}^{2}\right) q_{2}+\right. \\
& \left.+2 p_{2}\left(3 p_{1}^{2}+5 p_{2}^{2}+3 q_{1}^{2}\right) q_{2}^{2}+4 p_{1} q_{1} q_{2}^{3}+5 p_{2} q_{2}^{4}\right), \\
k_{8} & =\frac{1}{16}\left(4 p_{1}^{3} p_{2} q_{1}+p_{1}^{4} q_{2}+2 p_{1}^{2} q_{2}\left(3\left(p_{2}^{2}+q_{1}^{2}\right)+q_{2}^{2}\right)+4 p_{1} p_{2} q_{1}\left(p_{2}^{2}+q_{1}^{2}+3 q_{2}^{2}\right)+\right. \\
& \left.+q_{2}\left(5 p_{2}^{4}+5\left(q_{1}^{2}+q_{2}^{2}\right)^{2}+2 p_{2}^{2}\left(3 q_{1}^{2}+5 q_{2}^{2}\right)\right)\right) .
\end{aligned}
$$

As data to obtain estimates of the parameters of the mathematical model (1), we use a set of values $q_{1}=q_{1}\left(\omega_{0 k}\right), p_{1}=p_{1}\left(\omega_{0 k}\right), q_{2}=q_{2}\left(\omega_{0 k}\right), p_{2}=p_{2}\left(\omega_{0 k}\right)$ which are obtained from the experimental observation of the forced resonator oscillations excited with respect to the second fundamental mode with frequencies $\omega_{0}=\omega_{0 k}, k=\overline{1, N}$. The dependences of the stationary values $p_{1}, q_{1}, p_{2}, q_{2}$ on the frequency of oscillation excitation are shown in Fig. 2.

Amplitude-frequency characteristics $A=A\left(\omega_{0}\right)$ and $B=B\left(\omega_{0}\right)$, where $A=\sqrt{p_{1}^{2}+q_{1}^{2}}$ and $B=\sqrt{p_{2}^{2}+q_{2}^{2}}$, are shown in Fig. 3. The maximum value of the resonator oscillations amplitude is used for normalization. The relative oscillations amplitude A plotted on the vertical axis

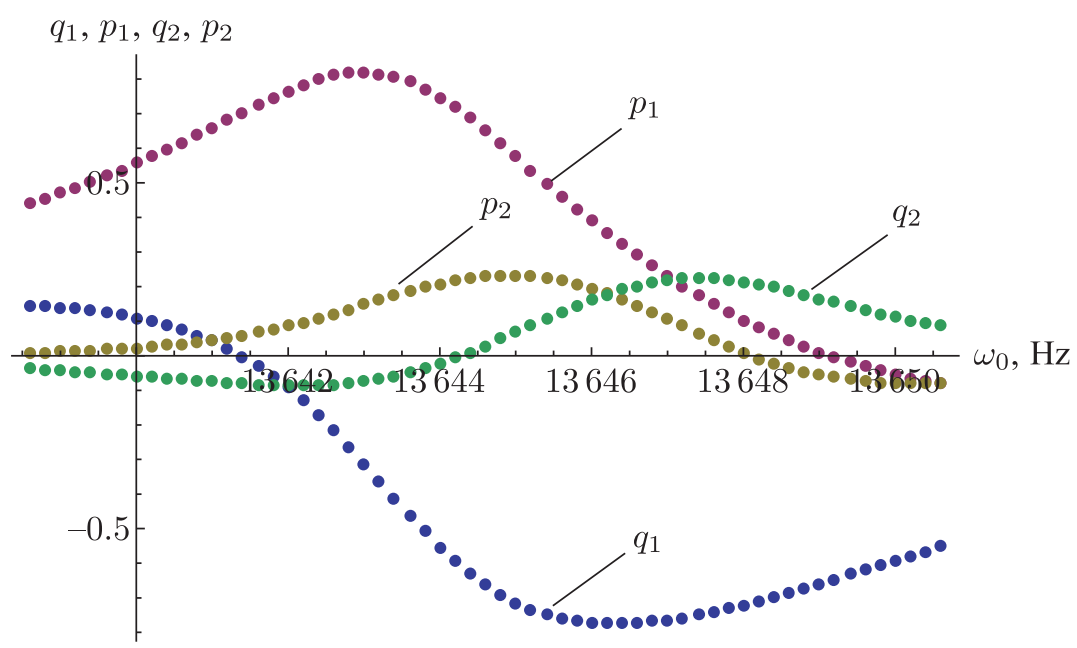

Fig. 2. Dependences of $p_{1}, q_{1}, p_{2}, q_{2}$ on the frequency of oscillation excitation. 


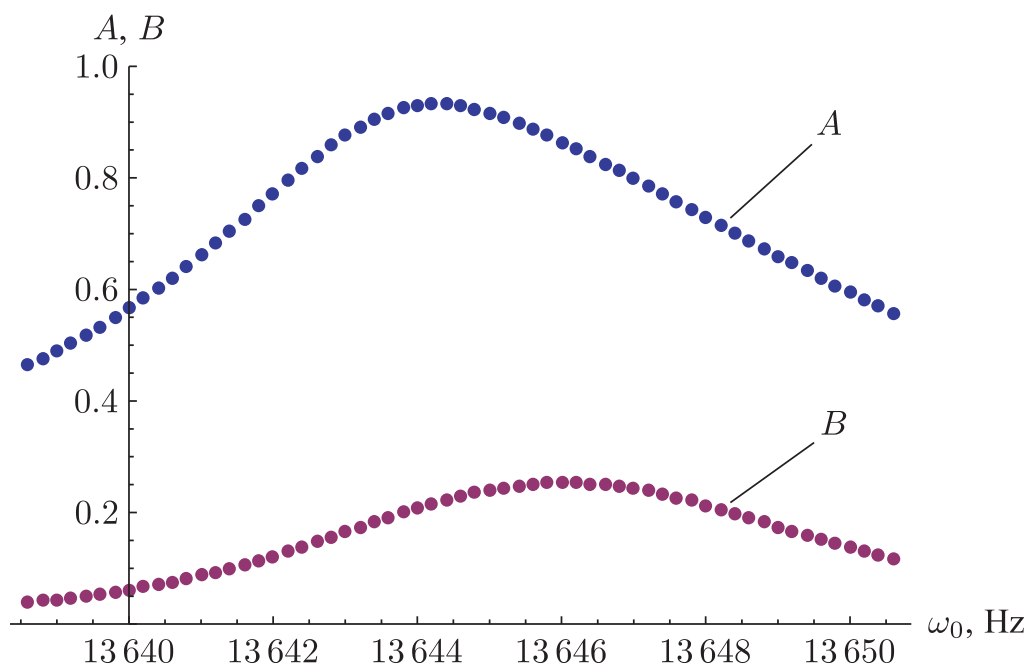

Fig. 3. Amplitude-frequency characteristics $A=A\left(\omega_{0}\right)$ and $B=B\left(\omega_{0}\right)$.

is equal to the ratio of the amplitude to the maximum value of the amplitude. The amplitudefrequency characteristic is known to be symmetric with respect to the resonant frequency in a small neighborhood of the resonance subject to linear oscillations. The symmetry of the amplitude-frequency characteristic $A=A\left(\omega_{0}\right)$ is disrupted. This means that oscillations are nonlinear.

To identify the parameters of Eqs. (3), we consider the stationary mode of forced resonator oscillations. Stationary oscillations are determined by the algebraic system of equations obtained from (3) with the value $\dot{\mathbf{q}}=\mathbf{0}$ and are represented with addition of the measurement error vector $\mathbf{e}_{\mathbf{j}}$ :

$$
\mathbf{y}_{j}=D_{j} \mathbf{z}+\mathbf{e}_{j}, \quad j=1, \ldots, N .
$$

Stationary values $p_{1}, q_{1}, p_{2}, q_{2}$ obtained with the given frequency adjustment $\lambda=\lambda_{j}$ in algebraic equations (4) are measured values, where $j=1, \ldots, N, N$ is the quantity of stationary modes of resonator oscillations.

Next, we set up an overdetermined algebraic system of equations with $N$ blocks (4) corresponding to the given frequency adjustments:

$$
\begin{gathered}
\mathbf{y}=D \mathbf{z}+\mathbf{e}, \\
D=\left(D_{1}^{T}, D_{2}^{T}, \ldots, D_{N}^{T}\right)^{T}, \quad \mathbf{y}=\left(\mathbf{y}_{1}^{T}, \mathbf{y}_{2}^{T}, \ldots, \mathbf{y}_{N}^{T}\right)^{T}, \quad \mathbf{e}=\left(\mathbf{e}_{1}^{T}, \mathbf{e}_{2}^{T}, \ldots, \mathbf{e}_{N}^{T}\right)^{T} .
\end{gathered}
$$

To identify the parameters of the system (5), it is sufficient to choose an array of values $\lambda_{j}$ $(j=1, \ldots, N)$ for which the matrix $C=\left(D^{T} D\right)^{-1}$ is not degenerate. By using the least squares method, we obtain the estimate $\widehat{\mathbf{z}}$ of the parameters of the mathematical model

$$
\widehat{\mathbf{z}}=\left(D^{T} D\right)^{-1} D^{T} \mathbf{y}
$$

which minimize the sum of squared residuals $S=(\mathbf{y}-D \widehat{\mathbf{z}})^{T}(\mathbf{y}-D \widehat{\mathbf{z}})$ and the variance $\widehat{\sigma}^{2}=$ $=S /(n-k)$.

The above method of deriving estimates leads to solving the system containing $n=4 N$ equations and $k$ unknowns. In the first case (only cubic nonlinearity) $k=13$, and in the 
second case (cubic and fifth degree nonlinearities) $k=14$. Therefore, at least $N=4$ stationary oscillation modes are required for measurements.

For interval estimation we assume that the vector random variables $\mathbf{e}_{i}, i=1, \ldots, N$, obey the normal law of distribution $\mathbf{e}_{i} \sim N\left(\mathbf{0}, \sigma_{e}^{2} I\right)$ and for all experiments have zero mathematical expectation $M\left[\mathbf{e}_{i}\right]=\mathbf{0}$ and the same variance $D\left[\mathbf{e}_{i}\right]=\sigma_{e}^{2} I$, where $I$ is the identity matrix. Errors in different experiments do not depend on each other: $\operatorname{cov}\left[\mathbf{e}_{i} \mathbf{e}_{j}\right]=M\left[\mathbf{e}_{i} \mathbf{e}_{j}\right]=\mathbf{0}$, $i, j=1, \ldots, N, i \neq j$.

This is the problem of estimating the expectation of the normal law of distribution in the case of unknown variance. Confidence intervals are found by the formula [15]

$$
\widehat{z}_{j}-t_{p} \widehat{\sigma} \sqrt{c_{j j}} \leqslant z_{j} \leqslant \widehat{z}_{j}+t_{p} \widehat{\sigma} \sqrt{c_{j j}}, \quad j=1, \ldots, k,
$$

where $c_{j j}$ are the diagonal elements of the matrix $C=\left(D^{T} D\right)^{-1}, t_{p}$ is the quantile of the order $p=\left(1+P_{c}\right) / 2$ for the Student distribution law with $n-k$ degrees of freedom, and $P_{c}$ is the confidence coefficient. If $n-k$ is small, then $t_{p}$ is chosen from the Student distribution table. If $n-k>30$, then $t_{p}$ can be chosen from Laplace's function.

\section{Experimental data and its processing}

By using the least squares method for a set of values $q_{1}=q_{1}\left(\omega_{0 k}\right), p_{1}=p_{1}\left(\omega_{0 k}\right), q_{2}=q_{2}\left(\omega_{0 k}\right)$, $p_{2}=p_{2}\left(\omega_{0 k}\right)$ obtained as a result of the experimental observation of the forced stationary oscillations, excited with respect to the second fundamental mode with frequencies $\omega_{0}=\omega_{0 k}$, $k=\overline{1, N}$, the estimation $\widehat{z}$ of the mathematical model parameters is obtained. The dependence of the quantities $\widehat{q}_{1}=\widehat{q}_{1}\left(\omega_{0 k}\right), \widehat{p}_{1}=\widehat{p}_{1}\left(\omega_{0 k}\right), \widehat{q}_{2}=\widehat{q}_{2}\left(\omega_{0 k}\right), \widehat{p}_{2}=\widehat{p}_{2}\left(\omega_{0 k}\right)$ on the frequency of oscillation excitation is restored via the obtained parameters estimates by solving the system of nonlinear algebraic equations (4). Errors between the restored amplitudes and the experimental ones are calculated from the following formulas:

$$
\Delta A=\sqrt{p_{1}^{2}+q_{1}^{2}}-\sqrt{\widehat{p}_{1}^{2}+\widehat{q}_{1}^{2}}, \quad \Delta B=\sqrt{p_{2}^{2}+q_{2}^{2}}-\sqrt{\widehat{p}_{2}^{2}+\widehat{q}_{2}^{2}} .
$$

In the first case the results obtained with linear (where $F_{1}=F_{2}=0$ ) and nonlinear (where $\left.F_{1}=\xi\left(f^{2}+g^{2}\right) f, F_{2}=\xi\left(f^{2}+g^{2}\right) g\right)$ mathematical models are compared. The identification method for gyroscope parameters using the linear mathematical model is described in $[7,8,16]$. In the case where the linear model is used for identifying the parameters, the sum of squared residuals is $S=20.3$, and when the nonlinear model is used, it is equal to $S=1.98$.

We emphasize that taking into account the nonlinearity coefficient allows one to reduce the sum of squared amplitude residuals by almost an order. From comparison of the errors between the restored and experimental amplitudes (Figs. 4 and 5) it follows that, for large oscillation amplitudes, taking nonlinearity into account significantly improves the accuracy of parameters identification for gyroscopes.

In the second case (cubic and fifth degree nonlinearities of spherical type) the sum of squared residuals is $S=0.99$. Taking into account the fifth degree nonlinearity allows one to reduce the sum of squared amplitude residuals by half.

From comparison of the errors between the restored and experimental amplitudes (Figs. 5 and 6) it follows that, for large oscillation amplitudes, taking nonlinearity into account improves the accuracy of parameter identification for gyroscopes.

The results presented above can be used for gyroscope drift compensation [17, 18].

RUSSIAN JOURNAL OF NONLINEAR DYNAMICS, 2018, 14(3), 377-386 


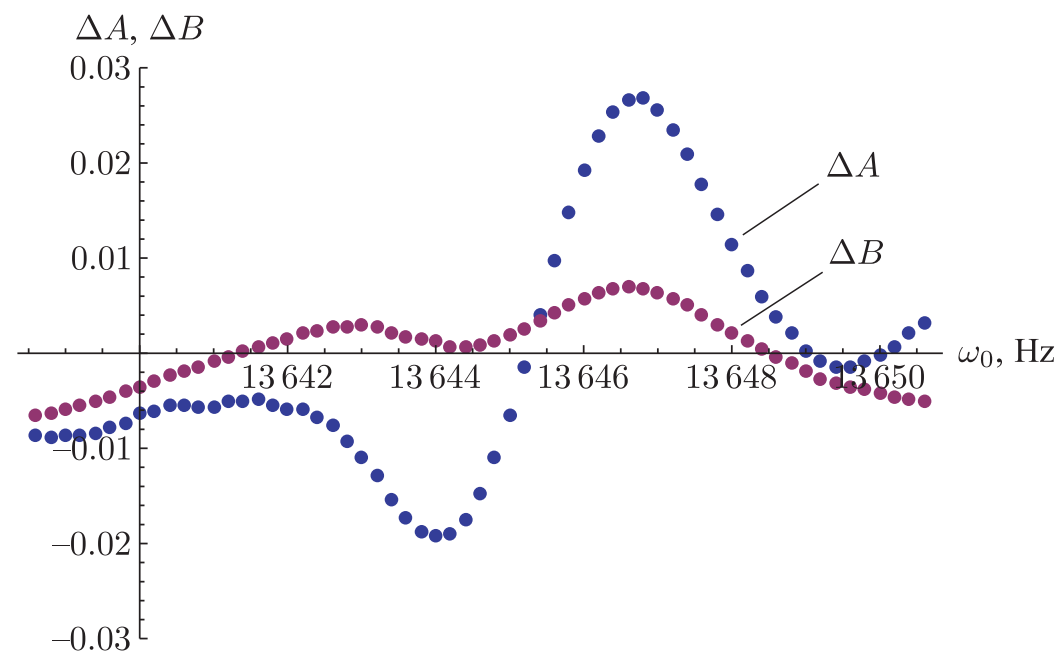

Fig. 4. Errors $\Delta A$ and $\Delta B$ between the restored and experimental amplitudes for the linear model.

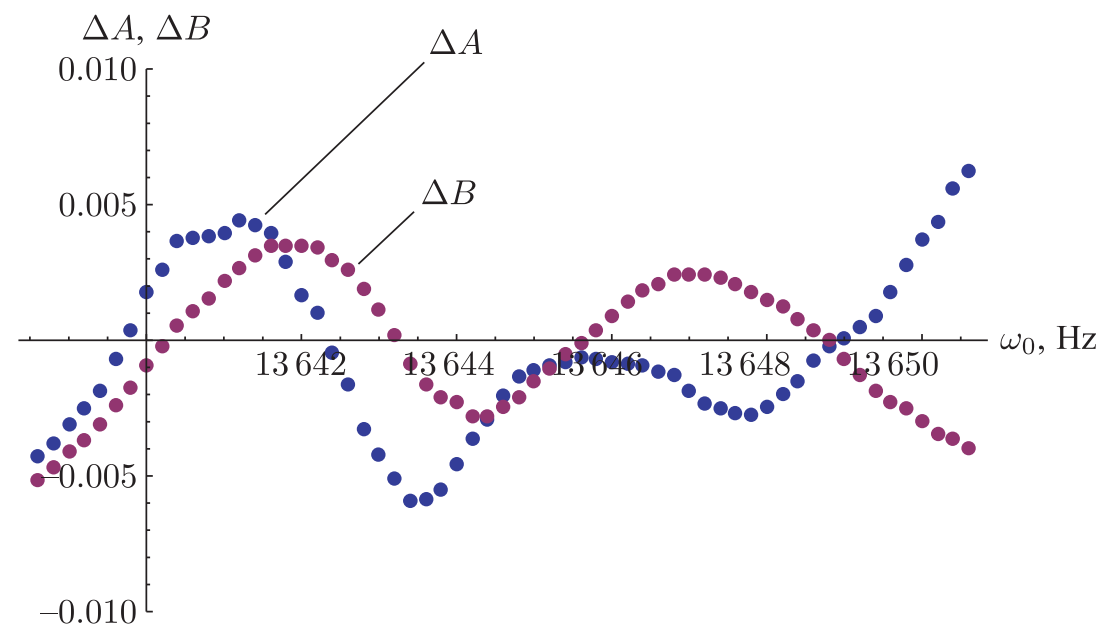

Fig. 5. Errors $\Delta A$ and $\Delta B$ between the restored and experimental amplitudes for the nonlinear model (with cubic nonlinearity).

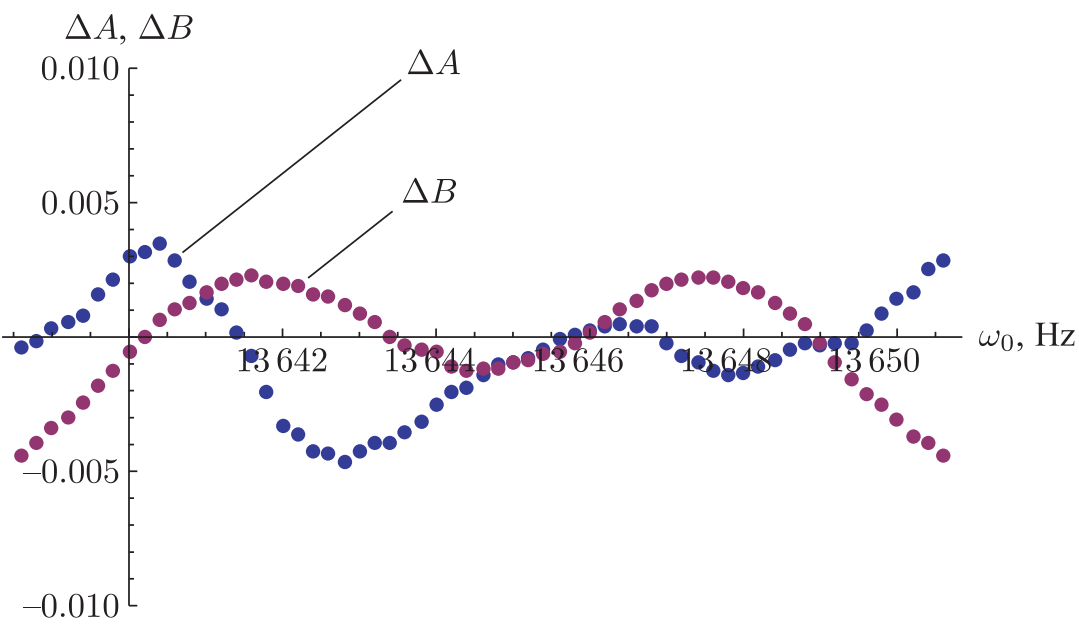

Fig. 6. Errors $\Delta A$ and $\Delta B$ between the restored and experimental amplitudes for the nonlinear model (with cubic and fifth degree nonlinearities). 


\section{Conclusions}

The parameter identification method for vibrating gyroscopes has been developed by using nonlinear terms in the mathematical model of resonator oscillations. This method allows testing at large oscillation amplitudes corresponding to a sufficiently high signal-to-noise ratio. The results of experimental data processing have shown that taking nonlinearities into account significantly increases the accuracy of parameter identification.

\section{References}

[1] Zhuravlev, V.F. and Klimov, D. M., Wave Solid State Gyroscope, Moscow: Nauka, 1985 (Russian).

[2] Zhuravlev, V.Ph., Theoretical Foundations of Wave Solid Gyroscope (WSG), Mech. Solids, 1993, vol. 28, no. 3, pp. 3-15; see also: Izv. Akad. Nauk. Mekh. Tverd. Tela, 1993, no. 3, pp. 15-26.

[3] Zhuravlev, V.Ph., The Controlled Foucault Pendulum As a Model of a Class of Free Gyros, Mech. Solids, 1997, vol.42, no.6, pp.21-28; see also: Izv. Akad. Nauk. Mekh. Tverd. Tela, 1997, no.6, pp. 27-35.

[4] Zhuravlev, V.F., The Task of Identification Errors of the Generalized Foucault Pendulum, Mech. Solids, 2000, no.5, pp.5-9; see also: Izv. Ross. Akad. Nauk. Mekh. Tverd. Tela, 2000, no.5, pp. 186-192.

[5] Zhbanov, Yu. K. and Zhuravlev, V.F., On Balancing of the Wave Solid State Gyroscope, Mech. Solids, 1998, vol.30, no.4, pp.851-859; see also: Izv. Akad. Nauk. Mekh. Tverd. Tela, 1998, no.4, pp. $4-16$.

[6] Matveyev, V.A., Lipatnikov, V.I., and Alekhin, A. V., Design of a Wave Solid-State Gyroscope, Moscow: MGTU, 1998 (Russian).

[7] Merkuryev, I. V. and Podalkov, V. V., Dynamics of the Micromechanical and Wave Solid-State Gyroscopes, Moscow: Fizmatlit, 2009 (Russian).

[8] Gavrilenko, A. B., Merkuryev, I. V., and Podalkov, V.V., Experimental Methods for the Determination Viscoelastic Anisotropy Parameters of the Wave Solid-State Gyroscope Resonator, Vestn. MPEI, 2010, vol. 15, no. 5, pp. 13-19 (Russian).

[9] De, S. K. and Aluru, N. R., Complex Nonlinear Oscillations in Electrostatically Actuated Microstructures, J. Microelectromech. Syst., 2006, vol. 15, no. 2, pp. 355-369.

[10] Rhoads, J. F., Shaw, S. W., Turner, K. L., Moehlis, J., DeMartini, B. E., and Zhang, W., Generalized Parametric Resonance in Electrostatically Actuated Microelectromechanical Oscillators, J. Sound Vibration, 2006, vol. 296, nos. 4-5, pp. 797-829.

[11] Chavarette, F. R., Balthazar, J. M., Guilherme, I. R., do Nascimento, O. S., and Peruzzi, N. J., A Reducing of Chaotic Behavior to a Periodic Orbit, of a Combdriver Drive System (MEMS) Using Particle Swarm Optimization, in Proc. of the 9th Brazilian Conf. on Dynamics Control and Their Applications (Serra Negra, 2010), pp. 378-383.

[12] Maslov, A. A., Maslov, D. A., and Merkurev, I. V., Parameter Identification of Hemispherical Resonator Gyro with the Nonlinearity of the Resonator, Pribory i Sistemy. Upravlenie, Kontrol, Diagnostika, 2014, no. 5, pp. 18-23 (Russian).

[13] Maslov, A. A., Maslov, D. A., and Merkuryev, I. V., Nonlinear Effects in Dynamics of Cylindrical Resonator of Wave Solid-State Gyro with Electrostatic Control System, Gyroscopy and Navigation, 2015, vol. 6, no. 3, pp. 224-229; see also: Giroskopiya i Navigatsiya, 2015, no. 1, pp. 71-80.

[14] Bogolubov, N. N. and Mitropolskiy, Yu. A., Asymptotic Methods in the Theory of Nonlinear Oscillations, Moscow: Nauka, 1974 (Russian).

[15] Ivchenko, G.I. and Medvedev, Yu. I., Introduction to Mathematical Statistics, 2nd ed., Moscow: URSS, 2017 (Russian). 
[16] Gavrilenko, A. B., Merkuryev, I. V., Podalkov, V.V., and Sbytova, E. S., Micromechanical Systems Dynamics, Moscow: MPEI, 2016 (Russian).

[17] Raspopov, V. Ya. and Yershov, R. V., Solid-State Wave Gyroscopes with Ring Resonator, Datchiki $i$ Sistemy, 2009, no. 5, pp.61-72 (Russian).

[18] Maslov, D. A. and Merkuryev, I. V., Compensation of Errors Taking into Account Nonlinear Oscillations of the Vibrating Ring Microgyroscope Operating in the Angular Velocity Sensor Mode, Nelin. Dinam., 2017, vol.13, no. 2, pp. 227-241 (Russian). 